\title{
Case of Anaphylaxis Induced by Rocuronium Treated with Sugammadex
}

\author{
Fabiano Timbó Barbosa, TSA 1, Rafael Martins da Cunha 2
}

\begin{abstract}
Summary: Barbosa FT, Cunha RM - Case of Anaphylaxis Induced by Rocuronium Treated with Sugammadex.
Background and objectives: Anaphylaxis during anesthesia is a rare event that may occur in up to $1: 20,000$ cases and approximately $60 \%$ to $70 \%$ of these cases are secondary to the use of muscle relaxants, particularly succinylcholine and rocuronium. The aim of this report is to describe the treatment of a case of rocuronium-induced anaphylaxis with inadequate response to traditional therapy, and the case resolution after using sugammadex.
\end{abstract}

Case report: A female patient, 62 years old, $72 \mathrm{~kg}$, was referred to the surgical center for treatment of epidural hematoma following an accident at home. She was monitored with pulse oximetry, cardioscope, and blood pressure meter for non-invasive blood pressure (BP) measurement. The baseline data were: $\mathrm{BP} 138 / 80 \mathrm{~mm} \mathrm{Hg}$, heart rate $(\mathrm{HR}) 80 \mathrm{bpm}$, and oxygen saturation $\left(\mathrm{SaO}_{2}\right) 100 \%$ on room air. Anesthesia was induced with propofol $70 \mathrm{mg}$, fentanyl $200 \mathrm{mg}$, and rocuronium $45 \mathrm{mg}$. After tracheal intubation, anaphylaxis non-responsive to conventional treatment was diagnosed. We chose the use of sugammadex $700 \mathrm{mg}$ intravenously as an adjuvant agent. The patient had reversal of the anaphylactic reactions with improvement of hemodynamic profile and was operated and conducted to the intensive care unit.

Conclusion: In this case, sugammadex was used to reverse the hemodynamic picture caused by rocuronium-induced anaphylaxis. However, it was not possible to identify the exact mechanism for the reversal.

Keywords: Anaphylaxis; Hypersensitivity; Neuromuscular Nondepolarizing Agents.

O2012 Elsevier Editora Ltda. All rights reserved.

\section{INTRODUCTION}

Anaphylaxis during anesthesia is a rare event that may occur in up to $1: 20,000$ cases and approximately $60 \%$ to $70 \%$ of these cases are secondary to the use of muscle relaxants, particularly succinylcholine and rocuronium ${ }^{1-3}$. The standard treatment requires immediate discontinuation of the drug, whenever possible, and the use of agents that improve cardiac performance and hemodynamic profile ${ }^{1}$.

Sugammadex was introduced into medical practice in order to antagonize the clinical action of rocuronium via encapsulation of this muscle relaxant, and this mechanism of action may contribute positively for the treatment of anaphylaxis induced by this neuromuscular blocker 4 .

The aim of this report is to describe the treatment of a case of rocuronium-induced anaphylaxis with inadequate response to traditional therapy and the case resolution after using sugammadex.

\footnotetext{
Received from Universidade Federal de Alagoas, Brazil.

1. Professor of Anesthesiology, Universidade Federal de Alagoas

2. Professor of Pharmacology, Centro de Ensinos Superiores de Maceió

Submitted on June 29, 2011

Approved on December 3, 2011.

Correspondence to:

Dr. Fabiano Timbó Barbosa

Rua Comendador Palmeira, 113, AP 202

Farol

57051150 - Maceió, AL, Brazil.

E-mail: fabianotimbo@yahoo.com.br
}

\section{CASE REPORT}

A female patient, 62 years old, $72 \mathrm{~kg}$, was referred to the surgical center for treatment of epidural hematoma following an accident at home. She denied allergies, systemic diseases, use of home medications, and complications in previous surgeries in which she received both neuraxial and general anesthesia in different procedures.

The patient was monitored with pulse oximetry, cardioscope, and blood pressure meter for non-invasive blood pressure (BP). Baseline data were: $\mathrm{BP} 138 / 80 \mathrm{~mm} \mathrm{Hg}$, heart rate (HR) $80 \mathrm{bpm}$, and oxygen saturation $\left(\mathrm{SaO}_{2}\right) 100 \%$ on room air. An 18G cannula was inserted into a peripheral vein and the anesthesia induced with propofol $70 \mathrm{mg}$, fentanyl $200 \mu \mathrm{g}$, and rocuronium $45 \mathrm{mg}$. No antibiotics were administered before or during anesthesia induction. The patient was orally intubated without presenting difficulties in airway management. Immediately after intubation, we noticed the appearance of erythematous plaques throughout the body and a probable allergic reaction was diagnosed. The patient's hemodynamic data after the reaction onset were: HR $145 \mathrm{bpm}$ with sinus rhythm, BP $80 / 60 \mathrm{~mm} \mathrm{Hg}$, and $\mathrm{SaO}_{2} 89 \%$ under $100 \%$ oxygen ventilation.

We started infusion of saline associated with doses of epinephrine $(0.30 \mathrm{mg})$ diluted in distilled water in a solution of $1: 10,000$ repeated every 5 minutes, and there was no clinical improvement after using adrenaline $(1.5 \mathrm{mg})$ and a crystalloid $(2,000 \mathrm{~mL})$.

We chose the use sugammadex $(700 \mathrm{mg})$ intravenously five minutes after rocuronium administration. After the use of sugammadex, we used an additional dose of $0.30 \mathrm{mg}$ of 
epinephrine and $500 \mathrm{~mL}$ of saline and saw a gradual decrease and disappearance of the plaques and hemodynamic improvement. The time for sugammadex response was 2 minutes. Hemodynamic data after response to sugammadex were HR $101 \mathrm{bpm}$ with sinus rhythm, BP 110/70 mm Hg, and $\mathrm{SaO}_{2} 99 \%$.

This was an emergency case without the possibility of postponing the procedure. Anesthesia was maintained with sevoflurane and $100 \%$ oxygen and local anesthetic infiltration in the scalp. There was no need for using vasopressors or incremental doses of epinephrine during the procedure. The patient was monitored with a neuromuscular junction monitor with the use of atracurium for the rest of the procedure without apparent signs of anaphylaxis. During surgery, the patient received hydrocortisone $500 \mathrm{mg}$ intravenously, and, after extubation, conducted to the intensive care unit. The patient consented to have her case reported in the scientific community.

\section{DISCUSSION}

Initially, susceptible individuals may be exposed to the antigen and produce IgE, which binds to receptors of mast cells and basophils ${ }^{5}$. When re-exposure to the antigen occurs, its binding with two IgE receptors induces tyrosine phosphorylation by tyrosine kinases ${ }^{5}$. A cascade of events begins and increases intracellular calcium with the release of histamine, tryptases, proteoglycans, and platelet-activating factor 5,6 . The phospholipid metabolism gives rise to leukotrienes (LTC4, LTD4, and LTE4) and prostaglandin D2 (PGD2) ${ }^{5}$. The set of histamines, PGD2 and LTC4, comprises potent agents to induce changes in capillary permeability, urticaria, erythema, angioedema, hypotension, and bronchospasm ${ }^{5}$. The anaphylactoid reaction results from the activation of the complement, cascade of bradykinin or direct activation of mast cells and basophils and is clinically indistinguishable from anaphylactic reaction ${ }^{5}$.

Anaphylaxis during anesthesia is a rare event occurring at a rate of $1: 3,500$ to $1: 20,000$ cases, being associated with significantly increased mortality ${ }^{5,7}$. Most cases have been associated with females and the use of muscle relaxants; however, latex and antibiotics also have a considerable number of cases nowadays ${ }^{7,8}$. Among the muscle relaxants, rocuronium has been implicated as the most commonly used agent, although this can be attributed to the greater use of the drug in our daily lives ${ }^{9}$.

Treatment measures for anaphylaxis seek to stabilize the patient and, among them, the withdrawal of the drug inducing the reaction is recommended ${ }^{1}$. Once the agent is adminis- tered intravenously, it is difficult to prevent the exposure and the reaction may be maintained until the body eliminates the drug after its metabolism ${ }^{4}$. Sugammadex was introduced into clinical practice to reverse the neuromuscular blockade induced by rocuronium. In contrast to the antagonistic agents, it encapsulates the drug molecule removing it from the circulation without acting competitively ${ }^{10}$. This mechanism of action of sugammadex may have potential positive action in the treatment of rocuronium-induced anaphylaxis, as it coats the portion of rocuronium that binds to $\lg$ E receptors ${ }^{4}$.

In this case report, sugammadex was used empirically in an attempt to generate a new molecule (rocuronium-sugammadex) and avoid drug exposure to the $\lg E$ receptors, if this were the mechanism of action. It is known that sugammadex does not encapsulate the whole molecule of rocuronium and, therefore, the part which binds to IgE receptors may not be prevented from binding to the antigen and may continue inducing the anaphylactic reaction 4,10 . Clinically, it is not possible to distinguish the cases as anaphylactic or anaphylactoid reaction; thus, it is not clear whether sugammadex should be used in all cases ${ }^{4}$. Further studies are needed to clarify in which situations one should employ the use of sugammadex ${ }^{1}$. In our case, the use of sugammadex was chosen due to the lack of patient's response to low doses of epinephrine and crystalloid infusion and because antibiotics and succinylcholine were not used during anesthetic induction.

The optimal dose of sugammadex for use in cases of anaphylaxis is not defined yet or in which conditions it should be used ${ }^{1}$. Some of the following questions remain: What is the optimal dose for anaphylaxis? What is the best time for administration? Should continuous infusion be used? What is the exact mechanism of action of sugammadex for these cases $^{1,4,10}$ ? The suggested dose of sugammadex for immediate reversal of neuromuscular blockade after administration of rocuronium $1 \mathrm{mg} \cdot \mathrm{kg}^{-1}$ is $16 \mathrm{mg} \cdot \mathrm{kg}^{-1}$ and, in cases of anaphylaxis, higher doses may be required ${ }^{4}$. In our case, the dose of $9.7 \mathrm{mg} \cdot \mathrm{kg}^{-1}$ of sugammadex $(700 \mathrm{mg}$ ) was given because it was the amount of drug available for use in the operating room at the time. Apparently, this dose of sugammadex completely reversed the clinical effect of rocuronium, taking also into account that it was not a low dose and it was administered approximately 25 minutes after the initial dose of rocuronium ${ }^{11}$. It is possible that restoration of muscle tone may contribute to the hemodynamic picture resolution, as reported in similar situation in the literature ${ }^{1}$.

To summarize, sugammadex was used in this case to reverse the hemodynamic picture of rocuronium-induced anaphylaxis. However, it was not possible to clarify the exact mechanism for the reversal. 


\section{REFERENCES}

1. McDonnell NJ, Pavy TJ, Green LK et al. - Sugammadex in the management of rocuronium-induced anaphylaxis. $\mathrm{Br} J$ Anaesth, 2011;106:199-201.

2. Harper NJ, Dixon T, Dugué $P$ et al. - Suspected anaphylactic reactions associated with anaesthesia. Anaesthesia, 2009;64:199-211.

3. Mertes PM, Laxenaire MC - Anaphylactic and anaphylactoid reactions occurring during anaesthesia in France. Seventh epidemiologic survey (January 2001-December 2002). Ann Fr Anesth Reanim, 2004;23:1133-1143.

4. Jones PM, Turkstra TP - Mitigation of rocuronium-induced anaphylaxis by sugammadex: the great unknown. Anaesthesia, 2010;65:89-90.

5. Hepner DL, Castells MC - Anaphylaxis during the perioperative period. Anesth Analg, 2003;97:1381-1395.

6. Koppert W, Blunk JA, Petersen LJ et al. - Different patterns of mast cell activation by muscle relaxants in human skin. Anesthesiology, 2001;95:659-667.

7. Fisher MM, Baldo BA - The incidence and clinical features of anaphylactic reactions during anesthesia in Australia. Ann Fr Anesth Reanim, 1993;12: 97-104.

8. Mertes PM, Laxenaire MC, Alla F - Anaphylactic and anaphylactoid reactions occurring during anesthesia in France in 1999-2000. Anesthesiology, 2003;99: 536-545.

9. Axon $A D$, Hunter JM - Editorial III: Anaphylaxis and anaesthesia-all clear now? Br J Anaesth, 2004;93: 501-504.

10. Naguib M - Sugammadex: another milestone in clinical neuromuscular pharmacology. Anesth Analg, 2007;104: 575-581.

11. Gijsenbergh $F$, Ramael S, Houwing $\mathrm{N}$ et al. - First human exposure of Org 25969, a novel agent to reverse the action of rocuronium bromide. Anesthesiology, 2005;103:695-703. 